\title{
Alkali-Metal-Mediated Zincation of Anisole: Synthesis and Structures of Three Instructive Ortho-Zincated Complexes
}

William Clegg, ${ }^{\dagger}$ Sophie H. Dale, ${ }^{\dagger}$ Allison M. Drummond, ${ }^{\star}$ Eva Hevia, ${ }^{\ddagger}$ Gordon W. Honeyman, ${ }^{\ddagger}$ and Robert E. Mulvey ${ }^{*}+$

WestCHEM, Department of Pure and Applied Chemistry, University of Strathclyde, Glasgow G1 1XL, U.K., and School of Natural Sciences (Chemistry), University of Newcastle, Newcastle upon Tyne NE1 7RU, U.K.

\section{Experimental Section}

General Methods. ${ }^{1} \mathrm{H},{ }^{13} \mathrm{C}$ NMR and ${ }^{7 \mathrm{Li}}$ spectra were recorded on a Bruker DPX 400 $\mathrm{MHz}$ spectrometer. All ${ }^{13} \mathrm{C}$ NMR spectra were proton decoupled. Hexane was distilled from sodium-benzophenone. $t$ Butyl lithium in hexane (Aldrich) was standardised immediately prior to use using salicylaldehyde phenylhydrazone. All synthetic work was carried out under an inert argon atmosphere.

Crystallographic data were collected on Bruker SMART and Nonius KappaCCD diffractometers at $150 \mathrm{~K}$, with $\operatorname{MoK\alpha }$ radiation $(\lambda=0.71073 \AA)$ and corrected semiempirically for absorption. All three structures are fully ordered; isotropic $\mathrm{H}$ atoms were constrained as riding, and other atoms were anisotropic. Crystal data for 2: $\mathrm{C}_{24} \mathrm{H}_{24} \mathrm{LiNO}_{2} \mathrm{Zn}, M=448.9$, monoclinic, space group $P 2_{1} / c, a=12.1007(18), b=$ 
11.1744(7), $c=19.3198(18) \AA, \beta=106.728(10)^{\circ}, V=2501.8(5) \AA^{3}, Z=4 ; 29018$ measured data, 5696 unique $\left(R_{\mathrm{int}}=0.045\right), R\left(\right.$ on $F$, for $\left.F^{2}>2 \sigma\right)=0.032, R_{\mathrm{w}}\left(\right.$ on all $\left.F^{2}\right)=$ 0.072, $S=1.02$, final difference map features within $\pm 0.36 \mathrm{e}^{-3}$. Crystal data for 3: $\mathrm{C}_{27} \mathrm{H}_{40} \mathrm{LiNO}_{3} \mathrm{Zn}, M=498.9$, monoclinic, space group $P 2_{1} / n, a=14.470(4), b=$ 11.4161(14), $c=16.167(4) \AA, \beta=93.35(2)^{\circ}, V=2666.1(10) \AA^{3}, Z=4 ; 34276$ measured data, 4644 unique $\left(R_{\text {int }}=0.083\right), R\left(\right.$ on $F$, for $\left.F^{2}>2 \sigma\right)=0.057, R_{\mathrm{w}}\left(\right.$ on all $\left.F^{2}\right)=0.094, S$ $=1.14$, final difference map features within $\pm 0.42 \mathrm{e}^{-3}$. Crystal data for 4 : $\mathrm{C}_{27} \mathrm{H}_{42} \mathrm{LiNO}_{2} \mathrm{Zn}, M=484.9$, triclinic, space group $P \overline{1}, a=10.399(2), b=11.205(2), c=$ 11.914(3) $\AA, \alpha=88.220(3), \beta=75.450(3), \gamma=87.077(3)^{\circ}, V=1341.7(5) \AA^{3}, Z=2 ; 9920$ measured data, 4693 unique $\left(R_{\mathrm{int}}=0.032\right), R\left(\right.$ on $F$, for $\left.F^{2}>2 \sigma\right)=0.053, R_{\mathrm{w}}\left(\right.$ on all $\left.F^{2}\right)=$ $0.105, S=1.25$, final difference map features within $\pm 0.73 \mathrm{e}^{-3}$ 


\section{Synthesis of $\left[(\mathrm{THF}) \mathrm{Li}(\mu-\mathrm{TMP})\left(\mu-\mathrm{C}_{6} \mathrm{H}_{4}-\mathrm{OMe}\right) \mathrm{Zn}(t \mathrm{Bu})\right](2)$}

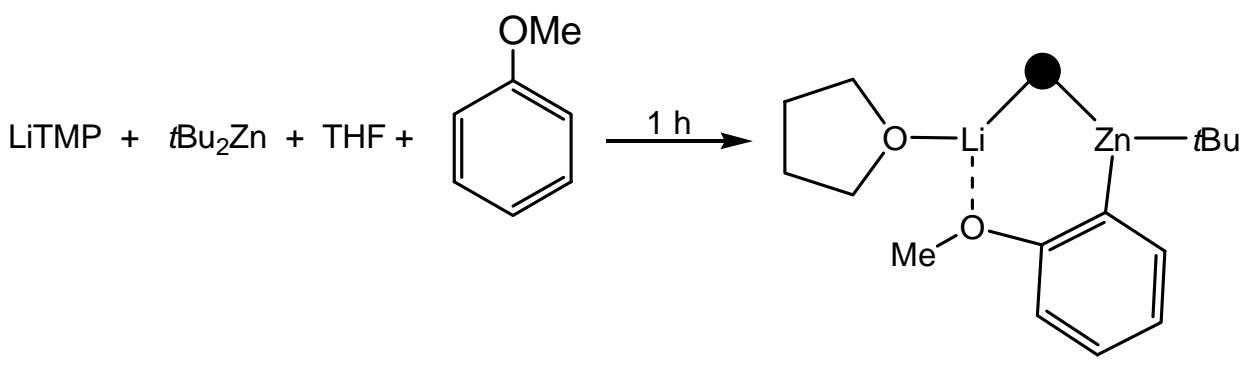

A solution of $t \mathrm{Bu}_{2} \mathrm{Zn}$ in hexane $(2 \mathrm{mmol})$ was added via canula to a solution of LiTMP in hexane [prepared in situ by reaction of BuLi $(1.25 \mathrm{~mL}$ of a commercial $1.6 \mathrm{M}$ solution in hexane, $2 \mathrm{mmol})$ and TMP(H) $(0.34 \mathrm{~mL}, 2 \mathrm{mmol})]$. THF $(0.16 \mathrm{~mL}, 2 \mathrm{mmol})$ was then introduced and the resulting colourless solution was allowed to stir for 10 minutes. Anisole $(0.22 \mathrm{~mL}, 2 \mathrm{mmol})$ was added and the mixture was stirred for 30 minutes. The volume of the solution was reduced by removing some solvent under vacuum. The resulting colourless solution was placed in the freezer at $-20^{\circ} \mathrm{C}$. A crop of colourless crystals was deposited after two days; one of them was employed in a X-ray diffraction experiment. $(0.63 \mathrm{~g}, 70 \%)$

${ }^{1} \mathrm{H}$ NMR $\left(\mathrm{C}_{6} \mathrm{D}_{6}\right):{ }^{*} 7.94\left(\mathrm{~d}, 1 \mathrm{H}, \mathrm{H}_{\text {meta }}\right), 7.22\left(\mathrm{t}, 1 \mathrm{H}, \mathrm{H}_{\text {para }}\right), 7.16\left(\mathrm{t}, 1 \mathrm{H}, \mathrm{H}_{\text {meta }}\right), 6.56(\mathrm{~d}$, meta $\left.1 \mathrm{H}, \mathrm{H}_{\text {ortho* }}\right), 3.31$ (s, 3H, OCH $\left.\mathrm{O}_{3}\right), 3.00$ (m, 4H, OCH $\left.\mathrm{O}_{2}, \mathrm{THF}\right), 1.96(\mathrm{~m}$, $\left.2 \mathrm{H}, H_{\gamma}, \mathrm{TMP}\right), 1.81\left(\mathrm{~m}, 4 \mathrm{H}, H_{\beta}, \mathrm{TMP}\right), 1.61\left(\mathrm{~s}, 9 \mathrm{H}, \mathrm{CH}_{3}, t \mathrm{Bu}\right), 1.56$ (s, 6H, $\left.\mathrm{CH}_{3}, \mathrm{TMP}\right), 1.27$ (s, 6H, $\left.\mathrm{CH}_{3}, \mathrm{TMP}\right), 1.17$ (m, 4H, $\mathrm{CH}_{2}$, THF).

${ }^{13} \mathrm{C}\left\{{ }^{1} \mathrm{H}\right\}$ NMR $\left(\mathrm{C}_{6} \mathrm{D}_{6}\right): 164.34\left(C_{\text {ipso }}\right), 150.38\left(\mathrm{Zn}-C_{\text {ortho }}\right), 142.54\left(C_{\text {meta }}\right), 127.12\left(C_{\text {meta }}\right)$, $123.37\left(C_{\text {para }}\right), 109.27\left(C_{\text {ortho* }}\right), 68.60\left(\mathrm{OCH}_{2}, \mathrm{THF}\right), 54.93\left(\mathrm{OCH}_{3}\right)$, $54.16\left(C_{\alpha}, \mathrm{TMP}\right), 40.95\left(C_{\beta}, \mathrm{TMP}\right), 35.44\left(\mathrm{CH}_{3}, t \mathrm{Bu}\right), 35.34\left(\mathrm{CH}_{3}\right.$, TMP), $35.14\left(\mathrm{CH}_{3}, \mathrm{TMP}\right), 25.46\left(\mathrm{CH}_{2}, \mathrm{THF}\right), 20.88\left(C\left(\mathrm{CH}_{3}\right)_{3}, t \mathrm{Bu}\right)$, $20.42\left(C_{\gamma}, \mathrm{TMP}\right)$.

${ }^{7} \mathrm{Li} \mathrm{NMR}\left(\mathrm{C}_{6} \mathrm{D}_{6}\right): 0.87$.

* The spectrum also showed the presence of traces of compound 3 . 
Synthesis of $\left[(\mathrm{THF}) \mathrm{Li}(\mu-\mathrm{TMP})\left(\mu-\mathrm{C}_{6} \mathrm{H}_{4}-\mathrm{OMe}\right) \mathrm{Zn}\left(\mathrm{C}_{6} \mathrm{H}_{4}-\mathrm{OMe}\right)\right](3)$

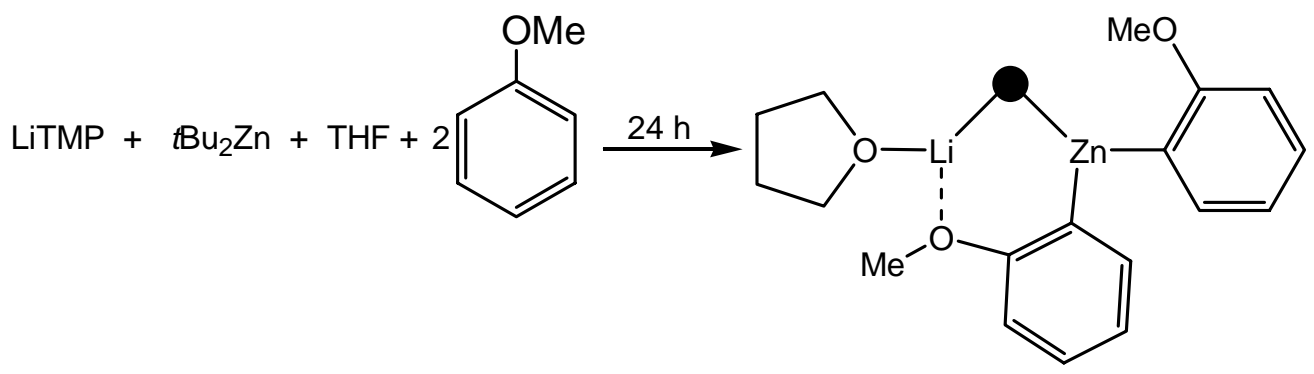

A solution of $t \mathrm{Bu}_{2} \mathrm{Zn}$ in hexane $(2 \mathrm{mmol})$ was added via canula to a solution of LiTMP in hexane [prepared in situ by reaction of BuLi (1.25 mL of a commercial 1.6M solution in hexane, 2mmol) and TMP(H) $(0.34 \mathrm{~mL}, 2 \mathrm{mmol})]$. THF $(0.16 \mathrm{~mL}, 2 \mathrm{mmol})$ was then introduced and the resulting colourless solution was allowed to stir for 10 minutes. Anisole $(0.44 \mathrm{~mL}, 4 \mathrm{mmol})$ was added and the mixture was stirred for 24 hours affording a slightly cloudy colourless solution which was placed in the freezer at $-20^{\circ} \mathrm{C}$. A crop of colourless crystals was deposited after two days, one of them was employed in an X-ray diffraction experiment. $(0.40 \mathrm{~g}, 41 \%)$

${ }^{1} \mathrm{H}$ NMR $\left(\mathrm{C}_{6} \mathrm{D}_{6}\right): 8.08\left(\mathrm{~d}, 1 \mathrm{H}, \mathrm{H}_{\text {meta }}\right), 7.22\left(\mathrm{t}, 1 \mathrm{H}, \mathrm{H}_{\text {para }}\right), 7.15\left(\mathrm{t}, 1 \mathrm{H}, \mathrm{H}_{\text {meta }}\right), 6.79$ (d, $\left.1 \mathrm{H}, \mathrm{H}_{\text {ortho* }}\right), 3.40$ (s, 3H, OCH $\mathrm{O}_{3}, 3.08\left(\mathrm{~m}, 4 \mathrm{H}, \mathrm{OCH}_{2}, \mathrm{THF}\right), 1.80(\mathrm{~m}$, 2H, $H_{\gamma}, \mathrm{TMP}$ ), 1.61 (s, 6H, $\left.\mathrm{CH}_{3}, \mathrm{TMP}\right), 1.35$ (s, 6H, $\mathrm{CH}_{3}, \mathrm{TMP}$ ), 1.23 (m, 4H, $\left.H_{\beta}, \mathrm{TMP}\right), 1.10$ (m, 4H, $\left.\mathrm{CH}_{2}, \mathrm{THF}\right)$.

${ }^{13} \mathrm{C}\left\{{ }^{1} \mathrm{H}\right\}$ NMR $\left(\mathrm{C}_{6} \mathrm{D}_{6}\right): 165.24\left(C_{\text {ipso }}\right), 151.74\left(\mathrm{Zn}-C_{\text {ortho }}\right), 141.64\left(C_{\text {meta }}\right), 127.45\left(C_{\text {meta }}\right)$, $123.20\left(C_{\text {para }}\right), 109.37\left(C_{\text {ortho* }}\right), 68.89\left(\mathrm{OCH}_{2}, \mathrm{THF}\right), 55.62\left(\mathrm{OCH}_{3}\right)$, $54.40\left(C_{\alpha}, \mathrm{TMP}\right), 40.98$ ( $\left.C_{\beta}, \mathrm{TMP}\right), 36.56,33.45\left(\mathrm{CH}_{3}, \mathrm{TMP}\right), 25.72$ $\left(\mathrm{CH}_{2}, \mathrm{THF}\right), 20.61\left(C_{\gamma}, \mathrm{TMP}\right)$.

${ }^{7} \mathrm{Li} \operatorname{NMR}\left(\mathrm{C}_{6} \mathrm{D}_{6}\right): 1.08$. 


\section{Synthesis of $\left[\left(\mathrm{C}_{6} \mathrm{H}_{5}-\mathrm{o}-\mathrm{OMe}\right) \mathrm{Li}(\mu-\mathrm{TMP})\left(\mu-\mathrm{C}_{6} \mathrm{H}_{4}-\mathrm{OMe}\right) \mathrm{Zn}(t \mathrm{Bu})\right](4)$}

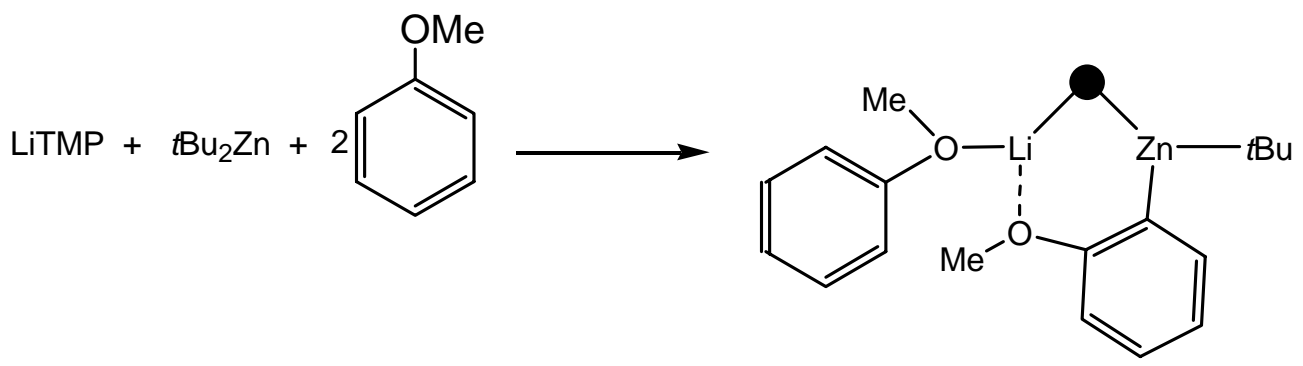

A solution of $t \mathrm{Bu}_{2} \mathrm{Zn}$ in hexane $(2 \mathrm{mmol})$ was added via canula to a solution of LiTMP in hexane [prepared in situ by reaction of BuLi $(1.25 \mathrm{~mL}$ of a commercial $1.6 \mathrm{M}$ solution in hexane, $2 \mathrm{mmol})$ and TMP(H) $(0.34 \mathrm{~mL}, 2 \mathrm{mmol})]$. Anisole $(0.44 \mathrm{~mL}, 4 \mathrm{mmol})$ was then added and the mixture was stirred at room temperature for one hour. The volume of the solution was reduced by removing some solvent under vacuum. The resulting colourless solution was placed in the freezer at $-20^{\circ} \mathrm{C}$. A crop of colourless crystals was deposited after two days. $(0.48 \mathrm{~g}, 34 \%)$. When this reaction was carried out using one molar equivalent of anisole $(0.22 \mathrm{~mL}, 2 \mathrm{mmol})$ following the same procedure as described above compound $\mathbf{4}$ was obtained as colorless crystals in a much lower yield $(0.17 \mathrm{~g}, 12 \%)$. The ${ }^{1} \mathrm{H}$ NMR spectrum of these crystals also showed the presence of traces of unreacted LiTMP.
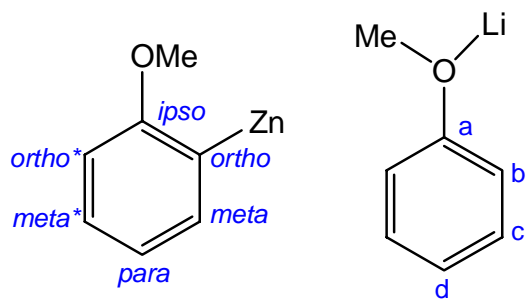

${ }^{1} \mathrm{H}$ NMR $\left(\mathrm{C}_{6} \mathrm{D}_{6}\right): 7.87\left(\mathrm{~d}, 1 \mathrm{H}, \mathrm{H}_{\text {meta }}\right), 7.10\left(\mathrm{~m}, 2 \mathrm{H}, \mathrm{H}_{\text {para }}\right.$ and $\left.\mathrm{H}_{\text {meta }}\right), 6.99\left(\mathrm{t}, 2 \mathrm{H}, H_{c}\right.$, anisole), 6.78 (d, $2 \mathrm{H}, H_{d}$, anisole), $6.51\left(\mathrm{~d}, 2 \mathrm{H}, H_{b}\right.$, anisole), $6.36(\mathrm{~d}$, $\left.1 \mathrm{H}, \mathrm{H}_{\text {ortho }}\right), 3.09\left(\mathrm{~s}, 3 \mathrm{H}, \mathrm{OCH}_{3}\right), 3.04\left(\mathrm{~s}, 3 \mathrm{H}, \mathrm{OCH}_{3}\right), 1.81(\mathrm{~m}, 2 \mathrm{H}$, $\left.H_{\gamma}, \mathrm{TMP}\right), 1.70\left(\mathrm{~m}, 4 \mathrm{H}, H_{\beta}, \mathrm{TMP}\right), 1.52$ (s, 9H, $\left.\mathrm{CH}_{3}, t \mathrm{Bu}\right), 1.39$ (s, 6H, $\left.\mathrm{CH}_{3}, \mathrm{TMP}\right), 1.21$ (s, 6H, $\mathrm{CH}_{3}, \mathrm{TMP}$ ). 
${ }^{13} \mathrm{C}\left\{{ }^{1} \mathrm{H}\right\}$ NMR $\left(\mathrm{C}_{6} \mathrm{D}_{6}\right): 164.21,158.26\left(C_{a}\right.$ and $\left.C_{i p s o}\right), 149.56\left(\mathrm{Zn}-C_{\text {ortho }}\right), 142.92\left(C_{\text {meta }}\right)$, $130.32\left(C_{d}\right), 128.30\left(C_{\text {meta }}\right), 123.66\left(C_{\text {para }}\right), 123.29\left(C_{c}\right), 115.51\left(C_{b}\right)$, $109.91\left(C_{\text {ortho* }}\right), 57.15,55.33\left(\mathrm{OCH}_{3}\right.$ 's $), 53.41\left(C_{\alpha}, \mathrm{TMP}\right), 40.69\left(C_{\beta}\right.$, TMP), $35.59\left(\mathrm{CH}_{3}, \mathrm{TMP}\right), 35.42\left(\mathrm{CH}_{3}, t \mathrm{Bu}\right), 34.99\left(\mathrm{CH}_{3}, \mathrm{TMP}\right)$, $20.96\left(C\left(\mathrm{CH}_{3}\right)_{3}, t \mathrm{Bu}\right), 20.26\left(C_{\gamma}, \mathrm{TMP}\right)$.

${ }^{7} \operatorname{Li~NMR}\left(\mathrm{C}_{6} \mathrm{D}_{6}\right): 1.01$.

\section{Conversion of Complex 4 into Complex 2 and then to 3}

Isolated crystals of $\mathbf{4}$ were dissolved in $\mathrm{C}_{6} \mathrm{D}_{6}$ solution to which were added two drops of $\mathrm{D}_{8}$-THF solution. The ${ }^{1} \mathrm{H}$ NMR spectrum of this solution revealed the presence of complex 2 and free anisole and the complete absence of complex 4.

In another experiment a hexane solution of $\mathbf{4}$ was treated with one molar equivalent of THF and the resulting solution was stirred for 24 hours. Crystals were deposited from this solution, which gave an identical NMR spectrum to that of complex $\mathbf{3}$. 